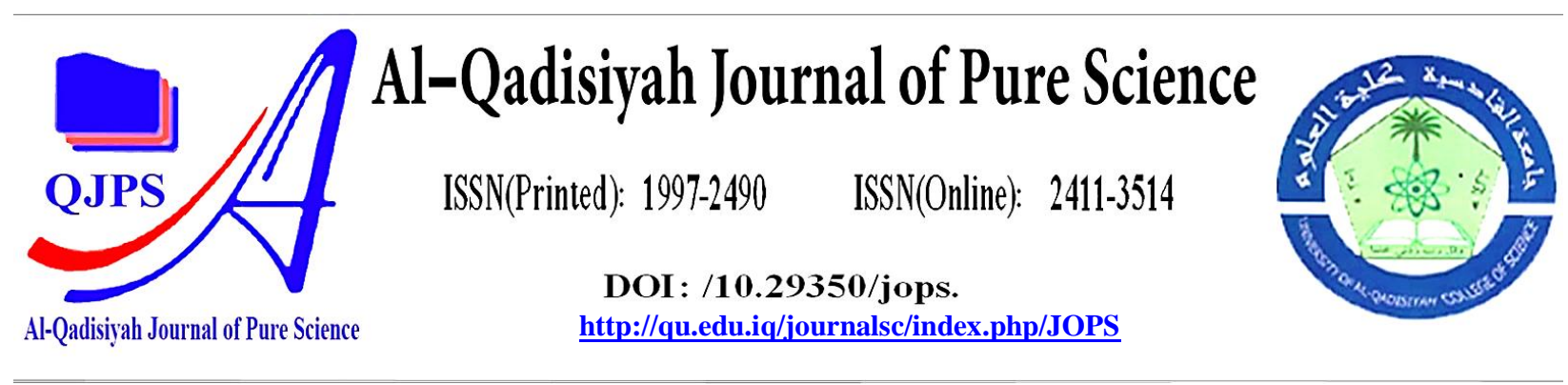

\title{
Design A Multi Biometric System for Safe Access to Buildings
}

\begin{tabular}{|c|c|}
\hline Authors Names & ABSTRACT \\
\hline $\begin{array}{l}\text { a.Shaymaa M. Hamandi } \\
\text { b.Abdul Monem S. Rahma } \\
\text { c.Rehab F. Hassan } \\
\text { Article History } \\
\text { Received on:6/7/2021 } \\
\text { Revised on: 30/7/2021 } \\
\text { Accepted on: 5/8/2021 } \\
\text { Keywords: } \\
\text { Multi Biometric, } \\
\text { Convolution Neural Network, } \\
\text { Thermal Imaging. } \\
\text { Passive Security, } \\
\text { CovID-19. } \\
\text { DOI: https://doi.org/10.29350/ } \\
\text { jops.2021.26. } 4.1341\end{array}$ & $\begin{array}{l}\text { Designing a multi-biometric system for safe access to buildings is a very } \\
\text { critical process because this system will act as an alternative of humans in } \\
\text { observation, and must make smart decisions to protect the building from any intruder } \\
\text { depending on the biometric information of the entering persons. In this paper a full } \\
\text { design of a proposed multi-biometric system will be presented, two sensors are used } \\
\text { (Visible and Thermal). the proposed system consists of several subsystems The first is } \\
\text { the construction of a database that contains all the photos and information about each } \\
\text { person who belongs to the building, the central system using visible and thermal } \\
\text { cameras, and the peripheral system using visible cameras only. The central system is } \\
\text { capable of remotely identifying and ensure of the health condition (Temperature, } \\
\text { Heart Rate, Respiration Rate) of each entering person which is a very important } \\
\text { process, especially with the COVID-19 pandemic. The peripheral system monitors } \\
\text { any suspicious issue like crowed or walking outside allowed lines. }\end{array}$ \\
\hline
\end{tabular}

\section{Introduction}

In now day's busy world, security is a major concern and everyone will look for reliable methods to guarantee safety [23], Biometric identification is a method of automatically recognizing or verifying human by physical features or personal attributes. The term "automatically" refers that biometric identification system must verify an individual feature's quickly with no interference from the user, which means passive authentication without knowledge of the examined person. The ideal biometric framework is one having the properties of peculiarity, inclusiveness, changelessness, agreeableness, collectability, and security [4]. Yet, there is no single biometric identifier that has these properties. As an answer, various biometric identifiers are utilized in a solitary framework which is usually known as a multimodal biometric framework. Because of solid and productive security arrangements in the security basic applications, multimodal biometric frameworks as of late arose in the biometric local area as an option in contrast to the conventional unimodal frameworks [22].

There are several types of biometric some are physiological that measure the physical characteristics of the human like (DNA, Ear, Face, Facial infrared thermogram, Fingerprint, palm print, Retina, Hand and finger geometry, and IRIS), and some are behavioral biometrics which based on measurements 
and data derived from action and indirectly measures characteristics of the human body like (Gate, Signature, voice, and Keystroke recognition).

The face, ear, facial infrared, and some behavioral biometrics are considered as passive security, while the other biometrics are considered as active security which needs a direct contact with the person.

This paper starts with reviewing the related works to the proposed system, definition and requirements of biometric systems and continues by describing biometric modal toward the multi-biometric systems' functionalities, structure and fusion methods.

\section{Related Work}

There are many design fusion methods using face identification to secure the entrance to a building or any private place, a lot of studies have been published to enhance the performance of the regular existent face biometric methods are discussed here.

Shaymaa Hamandi and et. El (2021) [8] have proposed a new multi-spectral hybrid invariant moments fusion technique for face identification, and employed Feed-forward neural network to train the moments' features and make decisions. The evaluation system uses databases of visible thermal pairs face images CARL and UTK-IRIS databases

Sánchez-Sánchez (2020) [25] have presented a multispectral examination of show assault discovery in robotized line control frameworks for facial biometrics utilizing the took in highlights from a convolutional neural organization, and utilized three sensors to plan and foster another information base that was made out of noticeable (VIS), close infrared (NIR), and warm pictures. Furthermore, five classifiers were utilized to identify the show assault. This examination demonstrated that warm sensors give preferable execution over different arrangements and the outcomes present better yields when all sensors are utilized together, whether or not classifier or highlight level combination was considered.

Arvind Jaiswal and Sandhya Tarar (2020) [11] have focused on the live security of public and private spots and products employments. What's more, utilized continuous security the IP CCTV cameras for observation purposes for face acknowledgment and distinguishing proof utilizing Local binary pattern histogram and Haar-cascade detection to track down the live area of the specific individual whenever and anyplace inside the city.

Mozammel Chowdhury (2020) [3] had proposed a multimodal biometric plot by consolidating the neighborhood highlights of face and ear biometrics in a computationally decent way and created calculations for face and ear acknowledgment by a combination of face and ear biometrics for human acknowledgment and confirmation. The trial assessment announced in this examination shows that the combination of these two (face and ear) biometrics results in a critical improvement in acknowledgment exactness contrasted with the precision accomplished by utilizing a singular one.

Qurban A. Memon (2020) [16] had proposed a diverse multimodal biometric approach utilizing three biometric strategies (finger impression, face, and voice) for cell phones. shown that the combination of biometric techniques can be layered to authorize private information security on a cell phone.

Bayram, K. S., \& Bolat, B. (2018) [2] have proposed a secure multi biometric framework, with various biometric modalities which are ear, face, and warm face. The face and warm face information were taken from USTC NVIE Spontaneous Database, while the ear information was gathered from IIT Delhi Ear Image Database. For every methodology, three element extraction strategies were utilized and four distinct classifiers (multi-layer perceptron, decision tree, support vector machines, and probabilistic neural network) were prepared by utilizing two combination techniques which were coordinating with score level and highlight level combination.

\section{Multi Biometric Identification system for passive Access Control}

The concept of passive security which is used in biometric approaches when there is a needing to identify or authorize anyone without he/she knowing to avoid the following: 
- Taking care when this person doesn't want anyone to know him (he may be a thief or an important man).

- Discomfit, many verifications may lead to stress nervous.

- Waiting for an instant of time to take the transition to identification or authorization systems. As mentioned about the importance of passive security (tracking) and verifying identity (without knowledge him/her to avoid putting him/her in a critical situation).

\subsection{Multi Biometric Identification System-Schemes}

As described in previous sections, a multimodal biometric framework is made by joining different unimodal frameworks. The data recovered in these individual frameworks is consolidated to make a multimodal framework. In such frameworks, the data can be consolidated through [19]:

- Multi-sensor, in this framework, a solitary biometric characteristic is imaged utilizing various sensors to remove data from enlisted pictures. For example, the face pictures of an individual are acquired utilizing a thermal infrared camera and an apparently visible camera [20].

- Multi-modal, these systems combine the evidence presented by different body traits for establishing identity. The cost of these systems is high since new sensors must be added [4].

- Multi-instance, these systems utilize various occasions of a similar body attribute. For instance, the left and right pointers. These frameworks are financially savvy since they require neither new sensors nor new calculations for highlight extraction [17].

- Multi-algorithm, in this system the biometric data is prepared by utilize various algorithms. This framework doesn't need the utilization of new sensors and hence is cost effective. For instance, a texture based algorithm and particulars put together calculation can work with respect to the same fingerprint picture [6].

- Multi-sample, in these systems one sensor is utilized to gain different samples to the same biometric trait. For example, face images, frontal face, left and right face.

The general approach to Multi-Biometric identification analysis consists of six main stages: (1) Data preprocessing, (2) Feature extraction, (3) Feature selection, (4) Multi-biometric fusion, (5) Template store in the database, and (6) classification. As shown in fig. 1.

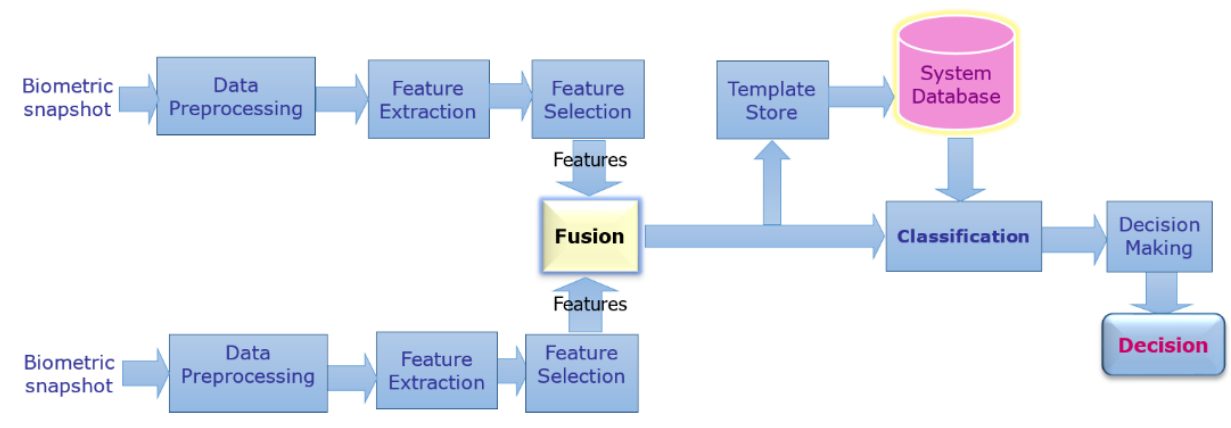

Fig. 1 - The main stages of multi-biometric system

\subsection{Multi Biometric Fusion}

- Fusion at Feature Level Fusion: at the feature level might be generally helpful for intently related modalities or for incorporating contribution from numerous sensors of the same methodology. It normalizes features from numerous sensors and remembers just for one vector, and afterward it chooses features utilizing some particular systems [10], like consecutive selection. It figures distance scores between an inquiry vector and one in the information base dependent on an action, for example, Euclidean distance [15]. 
- Fusion at Matching Level: Fusion at matching level standardizes scores of matchers to a similar area utilizing instruments like Min-Max, which maps score esteems to [0, 1], or Quadric-LineQuadric work, which attempts to isolate the genuine and impostor score distributions [13].

- Fusion at Decision Level Fusion of different biometrics done also at the decision level, which each classifier gives its decision as "accept" or "reject". After that the Borda count technique utilized for putting the classifiers' outcomes together.

\section{The Proposed System Design}

Single or unimodal biometric systems are not sufficient enough to identify a person efficiently so proposing a multi biometric system for identification and authentication to give a better reliable security. There will be particularly focusing on usage and rate of accuracy achieved by multi biometric identification systems for private and public security and various areas as in fig. 2 .

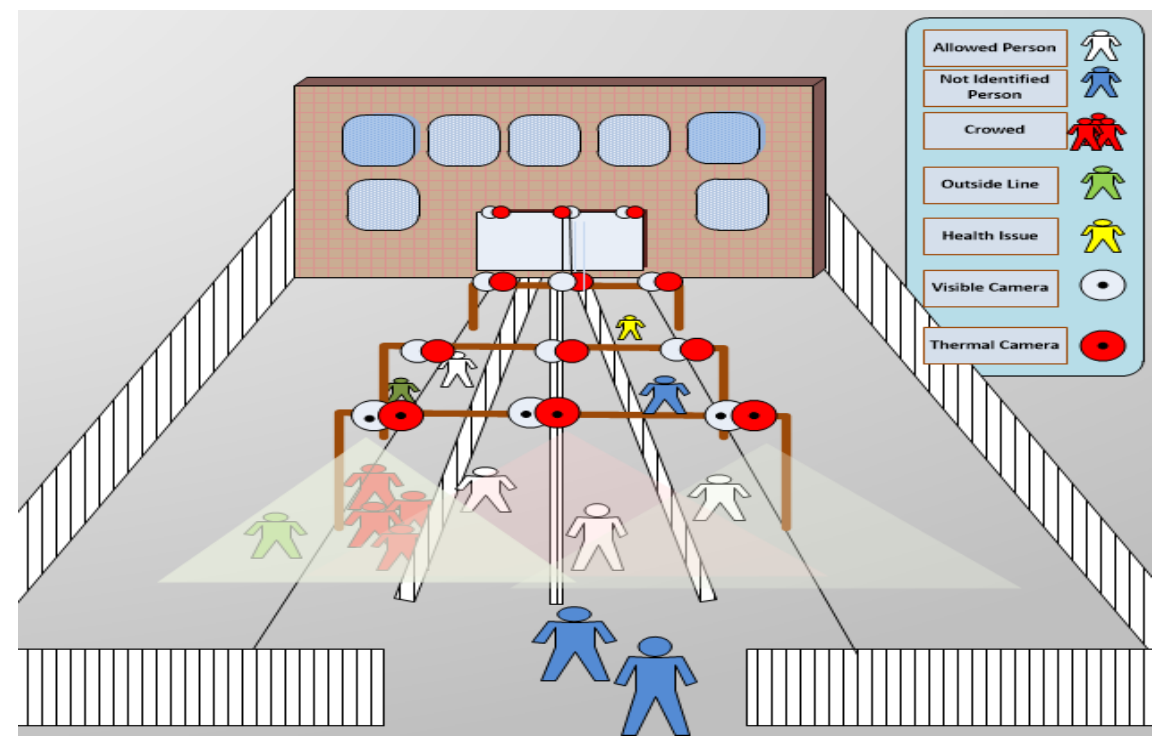

Fig.2 - Secure the entrance of building using multi biometric system

\subsection{Proposed System Components}

For multi-biometric system there will be a need for more than one sensor to provide a robust identification system.

1. Visible camera sensor: This sensor is capable of taking visible videos and stream it to the identification system.

2. Thermal camera sensor: This sensor is capable of taking thermal videos and stream it to the identification system.

3. Peripheral visible cameras: These camera sensors are capable of taking visible videos and stream it to the monitoring system.

4. Network communication system: The Network system must provide communication between the camera sensors and the proposed system, which involve the router and LAN or Wi-Fi connection. 


\subsection{The Proposed System Framework}

The proposed system consists of two subsystems as shown in fig. 3, The central and peripheral. The first is capable of processing both the thermal and visible camera video stream locate faces of the entering persons and make a decision if the person is allowed to enter the building or not according to the identification process and a health conformation process. The health conformation will be done automatically by the system.

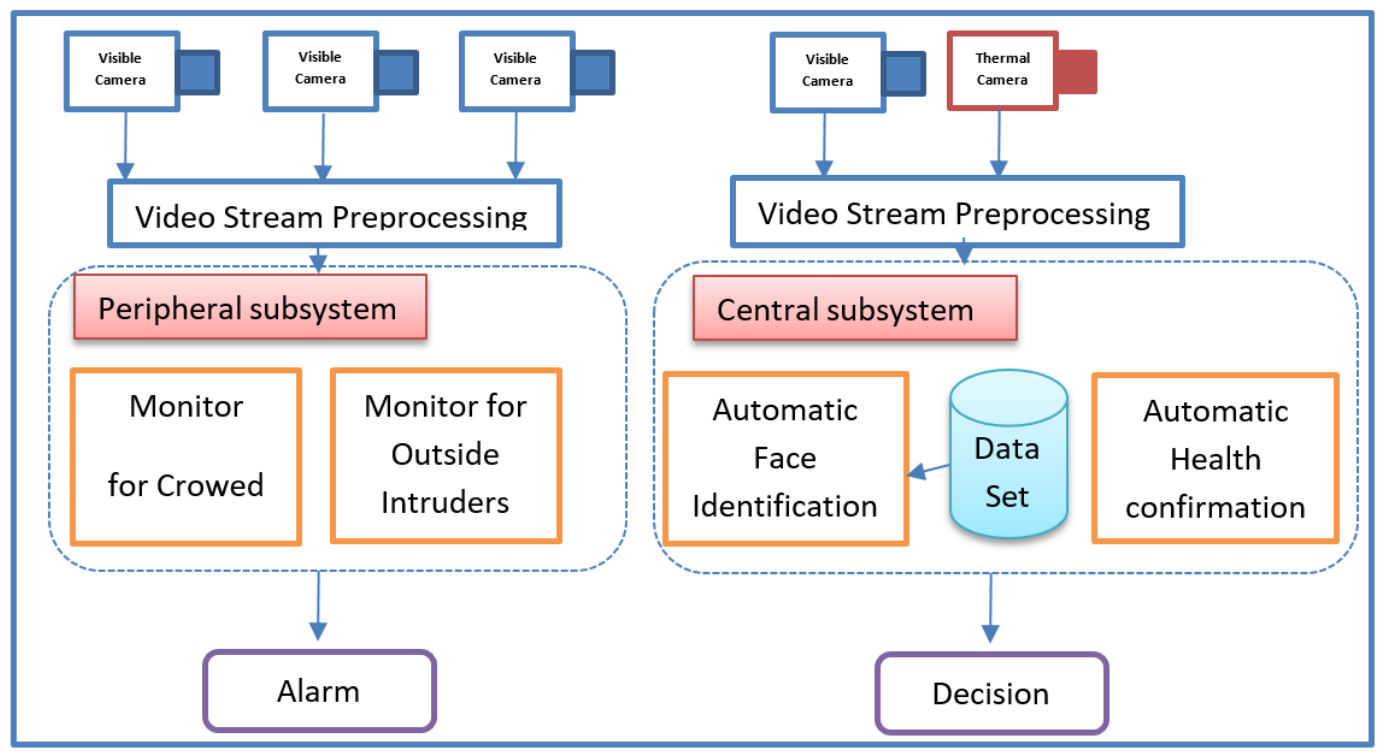

Fig. 3 The General Structure of the Proposed System

The second subsystem is capable of monitoring if there is a crowd of people or outside intruders and gives an alarm if there are any suspicious events.

The full processes of the proposed system are illustrated in the following flow chat in figure (4). 
Multi Biometric System for safe acess to building

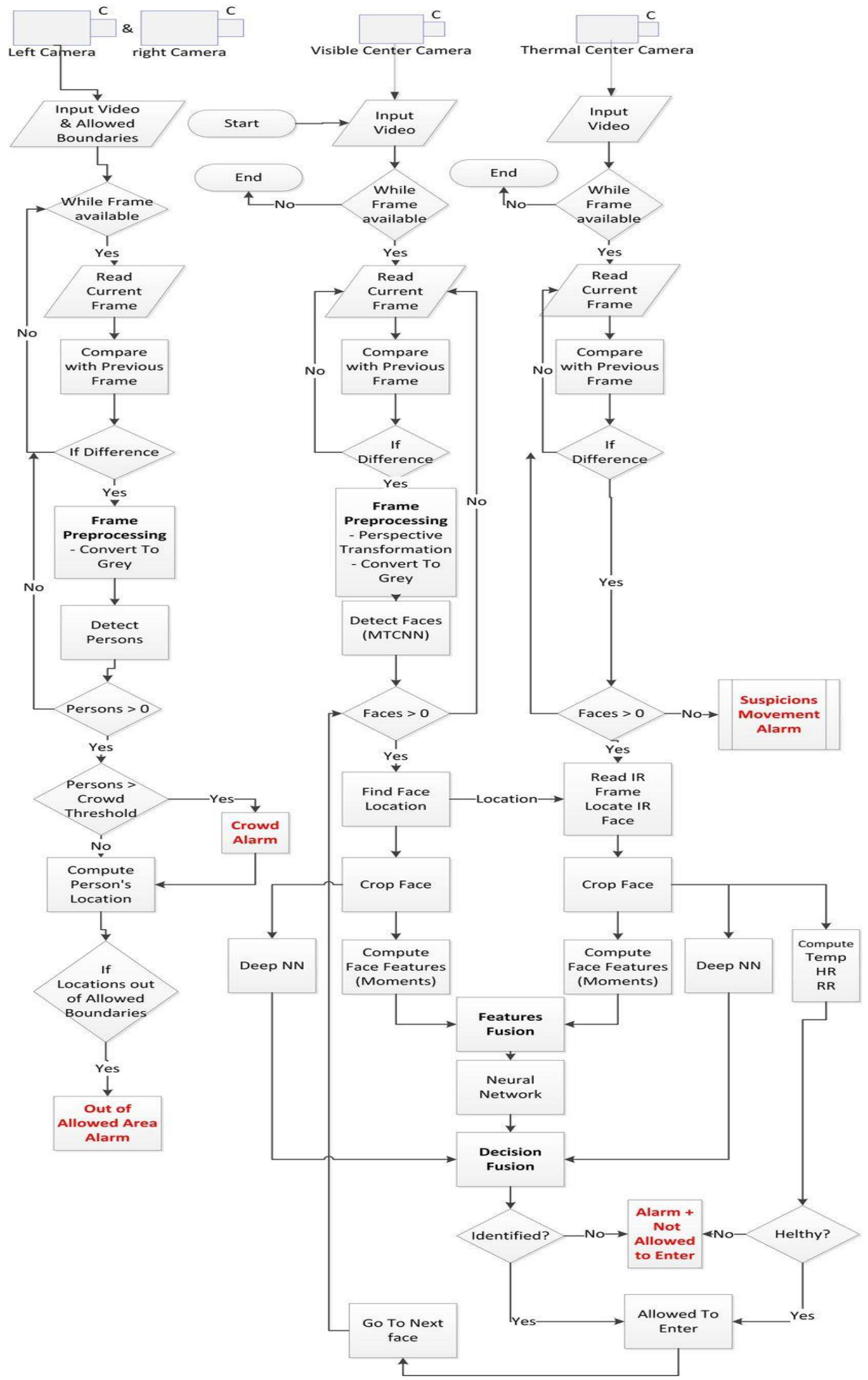

Fig. 4 - Flow chat presents the full processes of the proposed system 


\subsubsection{Camera video Streaming}

The first action is to capture the video frames from cameras and send these frames through TCP/IP connection to the target proposed system and while there is a difference between frames which means moving persons are in the scene; the frames are gone to the preprocessing stage.

If there is no difference between frames the system will just get the next frame.

\subsubsection{The Central Subsystem}

As shown in figure 3-1 this subsystem will take a video stream from two camera sensors (visible and thermal) and examine each person in the camera scene to give a decision whether this person is allowed to enter the building or not depending on the identification process of his face (Check existence in system dataset), and the ensure that this person is in a good health.

\subsubsection{Dataset Construction}

The proposed system allows only the persons who belong to the building to access, so there will be a need to a full data about each belonged person's Face (frontal, left, right, with glasses, with mask) and general information like (name, age, place, id, height, etc.) as shown in Fig 5.

This data will have stored in the dataset and processed to extract features which will trained in both neural network and deep neural network. The face of the person is recorded in multiple videos (visible and thermal) to gain the same face as seen from a camera, there will be no need for a preprocessing to the frames because the subjects will have captured in constrained environment.

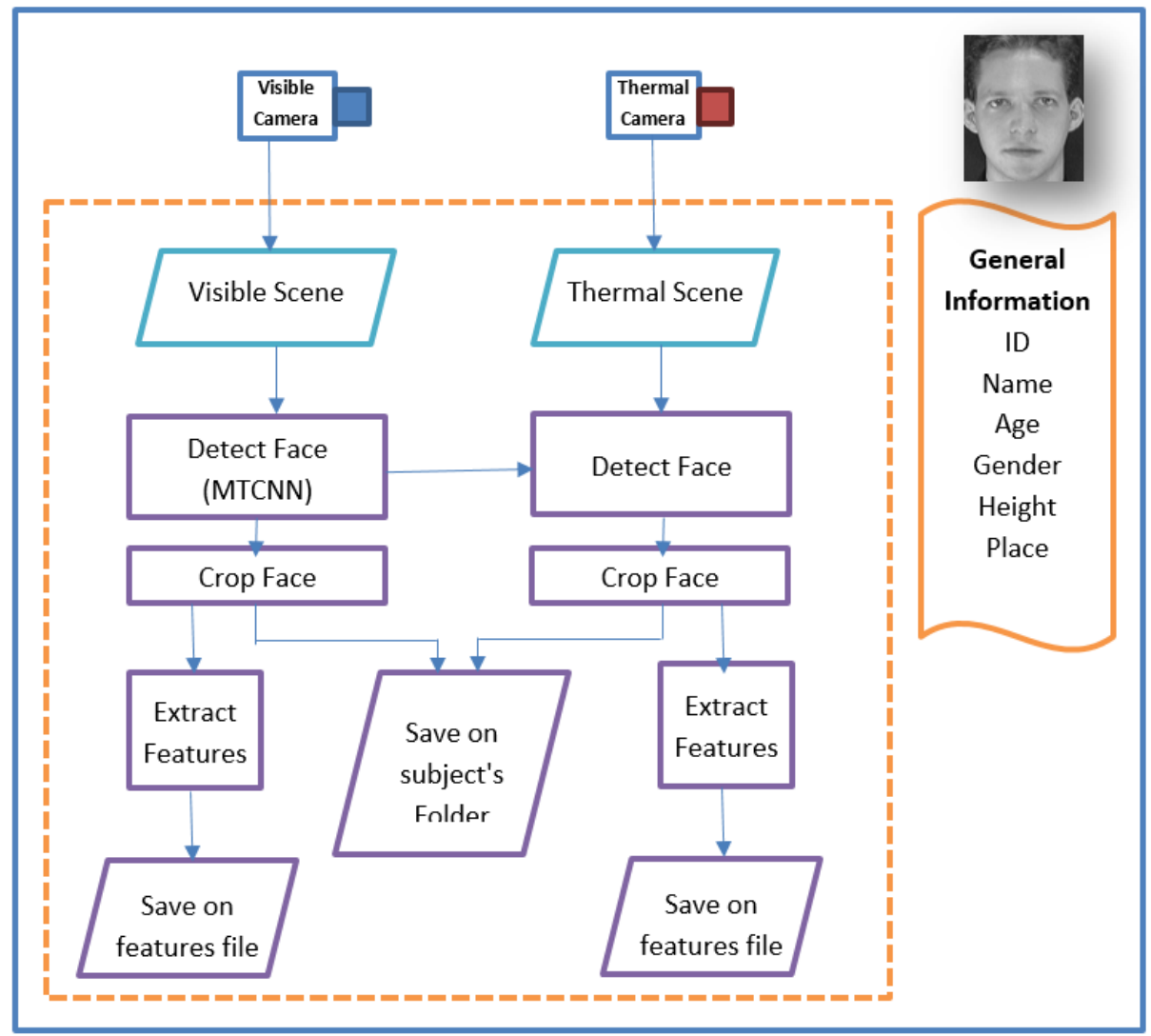

Fig.5 - Block diagram of dataset construction

The video sequences are recorded at different levels of zoom to explore the face resolution necessary to gain accurate facial analysis in multiple days. At end, the imaging scenarios utilized are intended to 
simulate an outdoor environment since the proposed system is designed to monitor the outside entrance of the building.

\subsection{Face Identification}

After faces were detected, their features will be extracted from more than one sensor (visible and thermal) and fused, then the faces are identified by more than one method (Feed-forward neural network and $\mathrm{CNN}$ ) and the decisions of these methods are fused to give a decision of if the person is allowed to access the building or not.

\subsubsection{Feed forward neural network Technique}

A feed-forward neural network is an artificial neural network wherein connections between the nodes do not form a cycle [9], in this network as shown in fig. 6, the data moves in one direction, only beginning with the input nodes, through the hidden nodes ending with the output nodes. There are no cycles in the network [26].

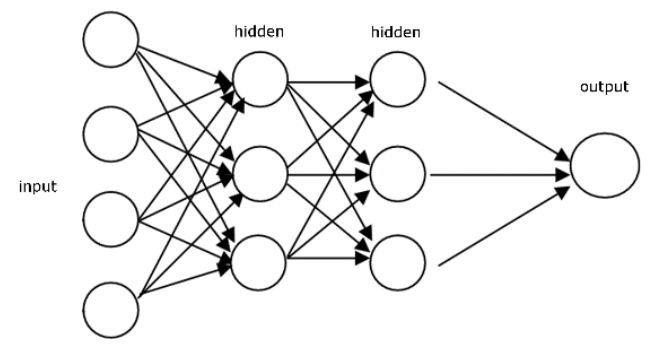

Fig.6 - Feed-forward neural network design.

\subsubsection{Deep Convolution Neural Network (CNN)}

The design of the CNN is based on AlexNet [1], and certain transformations to the current issue is done to improve the exhibition. The layers considered are:

- Convolutional Layer $(11 \times 11)+$ MaxPool layer $(2 \times 2)+$ Normalization layer

- Convolutional Layer $(4 \times 4)$

- Convolutional Layer $(3 \times 3)$

- Convolutional Layer $(3 \times 3)$

- Convolutional Layer $(3 \times 3)+$ MaxPool layer $(2 \times 2)$

- Dropout Layer

- Dropout Layer

- Fully connected Layer

The kernels' number utilized for each convolutional layer is 56 for layer number one, 156 for the layer number two, 256 for layer number three, 254 for layer number four, and 106 for the end convolutional layer. At the dropout layer, 2512 neurons were utilized, and 500 were fully connected (see Fig.7).

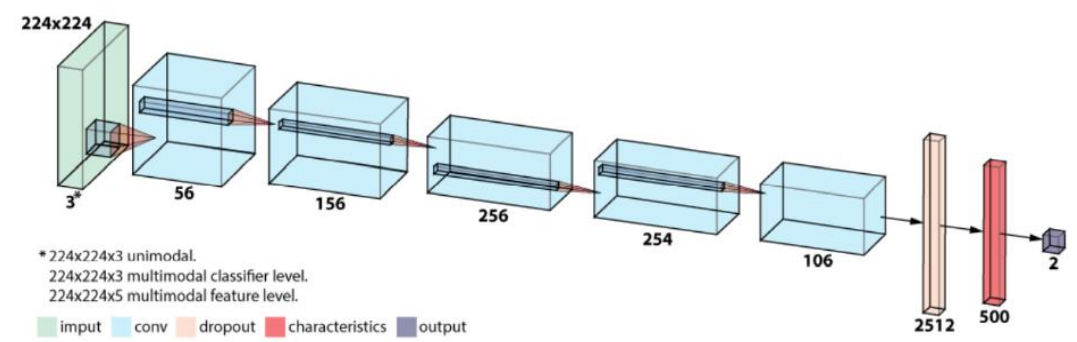

Fig 7-. CNN Architecture. 


\subsection{Biometric Security systems for HealthCare}

Anybody infected with disease appears on him with regular signs like fever, dry cough, weakness, and there are patients who feel a nasal blockage, or painful throat [7]. These signs could be diagnoses using two ways: the direct contact way and the contactless way. The contact technique has the benefits of being unconstrained by the environment and having a broad temperature range but utilizing an infrared-thermometer to compute the temperatures in public areas like an infrared-thermometer gun, have disadvantages also, which it cannot cover the whole people, it may aid the spreading of the disease, as the person who uses the infrared-thermometer gun has to be in a close range (five to fifteen centimeter) one by one person at a time, which is time-consuming and could lead to a large number of people queuing [7]. To avoid these drawbacks, another technology is needed. The accurate measurement of human body temperature at a distance becomes a key technology for mass health screening [5]. Thermal imaging is a non-contact temperature measurement technology that can provide remote monitoring of the thermal distributions and attempt to identify a potentially infected human from crowds [24]. The general framework of the biometric system for healthcare is shown in fig. 8 which detects three symptoms from visible and thermal imaging (the temperature, respiration rate, and heart rate) and gives a decision about if the individual is infected with a disease or not.

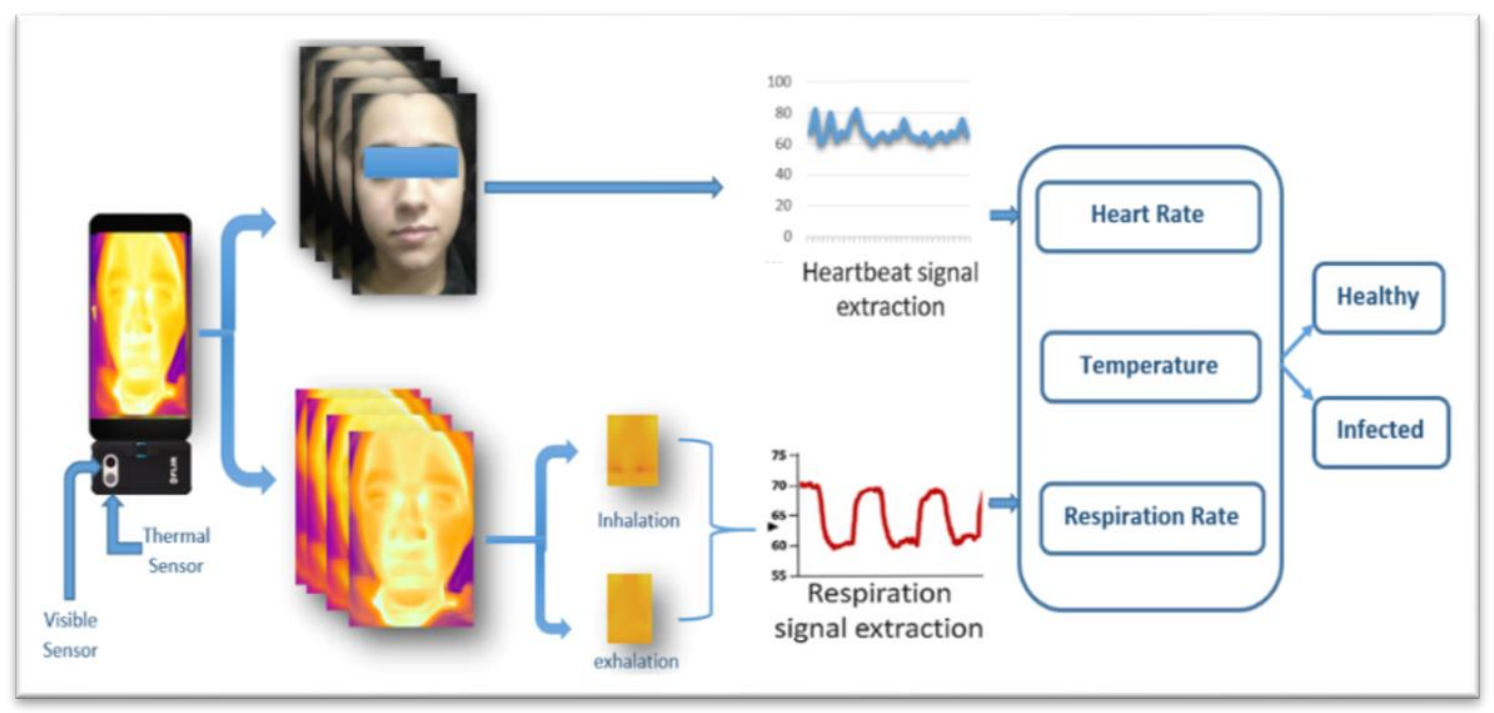

Fig. 8 - Framework of the biometric system for healthcare.

\subsubsection{Fever detection}

Infrared imaging has been proposed as a system for the detection of fever. As it measures the skin temperature, which is lower than the core body temperature, it is possible to detect fever. It is only necessary to establish an appropriate temperature baseline at, for example, the forehead. This will help to detect and defense from the screening of some diseases, like COVID-19, thus one of the first symptoms of a disease is a temperature change [27].

\subsubsection{Respiratory Rate Calculation}

Practice on thermography has confirmed it is reasonable to track respiration in a noncontact way using observing the temperature variations around the nostrils that are generated by inhalation and exhalation breathing cycles. By determining the nostril to be the region of interest and have checked the similarity between time-varying signals of the average temperature in the nostril-ROI to determine inhalation and exhalation. The method is based on tracking the nostril of the human and employing local temperature changes to indicate inhalation and exhalation cycles [28].

\subsubsection{Heart Rate Calculation}


Through the cardiac cycle, volumetric variations in the facial blood vessels change the amount of reflected ambient light absorption according to subsequent variations in the amount of ambient light. These variations show the timing of cardiovascular events. By streaming a video of the facial area with a camera, the red, green, and blue color sensors pick up a mixture of the reflected signals, including different sources of variations in light due to artefacts, heartbeats can be recognized by taking the brightness changes of RGB facial images made by variations in skin blood flow [21].

\section{Discussion}

The older style traditional methodologies like Personal Identification Number, password, key, login-id can be forgotten, taken or lost. In biometric authentication systems, a client doesn't have to remember any password or have a key. Like people perceive each other by actual appearance, biometric frameworks utilize actual attributes like fingerprints, facial recognition, iris to recognize a certifiable client and an intruder. At first biometric identification proof techniques were created to upgrade security in government and corporate areas, however these days it has advanced toward practically every one of the private areas like Banking. Money, Home wellbeing and security, Healthcare, Commercial security and security and so forth Since biometrics tests and formats of a biometric framework which has single biometric highlight to perceive a client can be supplanted and copied. In all the above cases because of bogus choices from the checking devices, an unapproved individual obtains entrance and it prompts fake movement. To defeat these issues, a biometric framework with a combination of at least two organic highlights and sensors called multi-biometric frameworks are utilized. The principal benefit of utilizing multimodal biometric acknowledgment is improved verification exactness. So utilizing multi-biometric acknowledgment frameworks help us in recognizing an individual all the more precisely when contrasted with utilizing a solitary biometric acknowledgment framework.

Table 1, presents the designs of some similar works and their difference from our work are reviewed.

\begin{tabular}{|c|c|c|c|c|c|}
\hline Author & Year & Study Title & Biometrics & Methodology & $\begin{array}{c}\text { Fusion } \\
\text { Type }\end{array}$ \\
\hline $\begin{array}{l}\text { Onur Can } \\
\text { KURBAN and } \\
\text { Ahmet BİLGIÇ } \\
{[14]}\end{array}$ & 2017 & $\begin{array}{l}\text { A Multi-Biometric } \\
\text { Recognition System } \\
\text { Based On Deep } \\
\text { Features of Face } \\
\text { and Gesture Energy } \\
\text { Image }\end{array}$ & $\begin{array}{l}\text { Face } \\
\text { Gesture } \\
\text { Energy }\end{array}$ & $\begin{array}{l}\text { deep learning features } \\
\text { with multiple light and } \\
\text { expression cases. }\end{array}$ & $\begin{array}{l}\text { Feature } \\
\text { Fusion }\end{array}$ \\
\hline $\begin{array}{l}\text { Kadir Sercan } \\
\text { Bayram and } \\
\text { Bülent Bolat [2] }\end{array}$ & 2018 & $\begin{array}{l}\text { Multi-biometric } \\
\text { identification by } \\
\text { using ear, face, and } \\
\text { thermal face }\end{array}$ & $\begin{array}{l}\text { Ear, face, } \\
\text { and thermal } \\
\text { face }\end{array}$ & $\begin{array}{l}\text { Three features } \\
\text { extraction methods are } \\
\text { utilized and four } \\
\text { classifiers (multilayer } \\
\text { perceptron, decision } \\
\text { tree, SVM, and } \\
\text { probabilistic NN) are } \\
\text { trained. }\end{array}$ & $\begin{array}{l}\text { Matching } \\
\text { score level } \\
\text { and } \\
\text { feature } \\
\text { level } \\
\text { fusion }\end{array}$ \\
\hline $\begin{array}{l}\text { A. H. Mohsin } \\
\text { et. El. [18] }\end{array}$ & 2018 & $\begin{array}{l}\text { Real-Time Remote } \\
\text { Health Monitoring } \\
\text { Systems Using } \\
\text { Body Sensor } \\
\text { Information and } \\
\text { Finger Vein } \\
\text { Biometric } \\
\text { Verification: A } \\
\text { Multi-Layer } \\
\text { Systematic Review }\end{array}$ & finger vein & $\begin{array}{l}\text { using a multi layer } \\
\text { comprehensive outline } \\
\text { of the sensor and } \\
\text { security in the } \\
\text { telemedicine } \\
\text { architecture as well as } \\
\text { finger vein biometric } \\
\text { verification systems in } \\
\text { the telemedicine } \\
\text { framework. }\end{array}$ & No Fusion \\
\hline
\end{tabular}




\begin{tabular}{|c|c|c|c|c|c|}
\hline $\begin{array}{l}\text { Edwin Jose, } \\
\text { Greeshma M and } \\
\text { Mithun Haridas } \\
\text { T. P [12] }\end{array}$ & 2019 & $\begin{array}{l}\text { Face Recognition } \\
\text { based Surveillance } \\
\text { System Using } \\
\text { FaceNet and } \\
\text { MTCNN on Jetson } \\
\text { TX2 }\end{array}$ & $\begin{array}{l}\text { Multiple } \\
\text { cameras }\end{array}$ & $\begin{array}{l}\text { capture frames using } \\
\text { OpenCV, detect faces } \\
\text { utilizing MTCNN, } \\
\text { faces Embedding with } \\
\text { triplet loss and Siamese } \\
\text { Network faces } \\
\text { identification by } \\
\text { FaceNet algorithm, } \\
\text { storing the recognized } \\
\text { face for analysis and to } \\
\text { monitor intruder alert. }\end{array}$ & \\
\hline $\begin{array}{l}\text { M. Araceli } \\
\text { Sánchez-Sánchez } \\
\text { and et. El [25] }\end{array}$ & 2020 & $\begin{array}{l}\text { CNN Approach for } \\
\text { Multi spectral } \\
\text { Facial Presentation } \\
\text { Attack Detection in } \\
\text { Automated Border } \\
\text { Control Systems }\end{array}$ & $\begin{array}{l}\text { visible, near } \\
\text { infrared, and } \\
\text { thermal } \\
\text { face's } \\
\text { pictures. }\end{array}$ & $\begin{array}{l}\text { multiple classifiers } \\
\text { were utilized (linear } \\
\text { and RBF SVM, KNN, } \\
\text { decision tree, and } \\
\text { logistic regression). }\end{array}$ & $\begin{array}{l}\text { Feature } \\
\text { Fusion }\end{array}$ \\
\hline $\begin{array}{l}\text { MOZAMMEL } \\
\text { CHOWDHURY } \\
{[3]}\end{array}$ & 2020 & $\begin{array}{l}\text { A Robust } \\
\text { Multimodal } \\
\text { Biometric Scheme } \\
\text { for Human } \\
\text { Recognition and } \\
\text { Authentication }\end{array}$ & face and ear & $\begin{array}{l}\text { For ear recognition, the } \\
\text { system detects and } \\
\text { extracts the ear region } \\
\text { from the facial image } \\
\text { geometry and then use } \\
\text { the ear features to } \\
\text { recognize the ear. }\end{array}$ & $\begin{array}{l}\text { Feature } \\
\text { fusion and } \\
\text { match } \\
\text { score level } \\
\text { fusion }\end{array}$ \\
\hline $\begin{array}{l}\text { Qurban A. } \\
\text { Memon [16] }\end{array}$ & 2020 & $\begin{array}{l}\text { Multi layered } \\
\text { Multimodal } \\
\text { Biometric } \\
\text { Authentication for } \\
\text { Smartphone } \\
\text { Devices }\end{array}$ & $\begin{array}{l}\text { fingerprint, } \\
\text { face and } \\
\text { voice }\end{array}$ & $\begin{array}{l}\text { adopted convenient } 3 \text { - } \\
\text { step (multi-layered) } \\
\text { multimodal processes, } \\
\text { SVM was utilized to } \\
\text { train and test the } \\
\text { network. }\end{array}$ & $\begin{array}{l}\text { score } \\
\text { based } \\
\text { fusion }\end{array}$ \\
\hline $\begin{array}{l}\text { Shaymaa } \\
\text { Hamandi and et } \\
\text { el. [8] }\end{array}$ & 2021 & $\begin{array}{l}\text { Multi-Spectral } \\
\text { Hybrid Invariant } \\
\text { Moments Fusion } \\
\text { Technique for Face } \\
\text { Identification }\end{array}$ & $\begin{array}{l}\text { Face } \\
\text { invariant } \\
\text { shape } \\
\text { moments } \\
\text { Visible and } \\
\text { thermal } \\
\text { sensors }\end{array}$ & $\begin{array}{l}\text { exploits the power of } \\
\text { thermal facial image } \\
\text { invariant moments } \\
\text { features fused with the } \\
\text { visible facial image } \\
\text { invariant moments } \\
\text { features. And employs } \\
\text { Feed-forward neural } \\
\text { network to train the } \\
\text { moments' features and } \\
\text { make decisions. }\end{array}$ & $\begin{array}{l}\text { Feature } \\
\text { fusion }\end{array}$ \\
\hline
\end{tabular}

Table 1 - Multi-Biometric Systems.

This study is distinguished from previous similar works in that it remotely monitors the health status of those entering the building as well as identifying their identity, which is one of the most important requirements of this period due to the spread of Covid-19 disease. Thus the protection of the building is fully integrated from any intruder and protects people in the building from any suspected illness.

\section{Conclusion}

The aim of this study is to propose a robust and smart design to protect the buildings automatically from the entry of unauthorized persons, as well as to check their health condition by measuring remotely person temperature, heart rate and breathing, in addition to monitoring any suspicious cases such as crowed or walk outside the entry line. More than one sensor (visible and thermal) was used, 
and the monitoring process was done at several levels to ensure more accuracy. At the beginning, faces were detected and their features were extracted from more than one sensor (visible and thermal), then the faces are identified by more than one method (Feed-forward neural network and CNN) and the decisions of these methods are fused to give a decision of if the person is allowed to access the building or not. This design system could be used to monitor the entrance of private buildings, companies, banks, and schools. Most investigations depend on lab or ideal conditions-controlled conditions. In any case, in a genuine situation, a subject's circumstance is totally adjusted because of assorted physiological conditions, like pressure, temperature changes, perspiring, and expanded pulse. Thus, the additional worth of this examination is that this information base was obtained in real time and at different days and times with real out door conditions which makes the result more reliable and close to reality.

\section{References}

[1] C. Alippi, S. Disabato, M. Roveri, "Moving convolutional neural networks to embedded systems: the alexnet and VGG-16 case", in: 2018 17th ACM/IEEE Int. Conf. Inf. Process. Sens. Networks, IEEE, 2018: pp. 212-223.

[2] K.S. Bayram, B. Bolat, "Multibiometric identification by using ear, face, and thermal face", EURASIP J. Image Video Process. 2018 pp. 1-8, 2018.

[3] M. CHOWDHURY, "A Robust Multimodal Biometric Scheme for Human Recognition and Authentication", 2020.

[4] W. Dahea, H.S. Fadewar, "Multimodal biometric system: A review", Int. J. Res. Adv. Eng. Technol. 4 pp. 25-31, 2018.

[5] D. Dias, J. Paulo Silva Cunha, "Wearable health devices — vital sign monitoring, systems and technologies", Sensors. 18 pp. 2414, 2018.

[6] L.M. Dinca, G.P. Hancke, "The fall of one, the rise of many: a survey on multi-biometric fusion methods", IEEE Access. 5 pp. 6247-6289, 2017.

[7] T. Greenhalgh, G.C.H. Koh, J. Car, "Covid-19: a remote assessment in primary care", Bmj. 368 2020.

[8] S. Hamandi, A.M. Rahma, R. Hassan, "Multi-Spectral Hybrid Invariant Moments Fusion Technique for Face Identification", Int. Arab J. Inf. Technol. 18 pp. 405-413, 2021.

[9] S.M. Hamandi, A.M.S. Rahma, R.F. Hassan, "A New Hybrid Shape Moment Invariant Techniques for Face Identification in Thermal and Visible Visions", in: 2020 21st Int. Arab Conf. Inf. Technol., IEEE, 2020: pp. 1-9.

[10] S.M. Hamandi, A.M.S. Rahma, R.F. Hassan, "A New Hybrid Technique for Face Identification Based on Facial Parts Moments Descriptors", Eng. Technol. J. 39 pp. 117-128, 2021.

[11] A. Jaiswal, S. Tarar, "Real-Time Biometric System for Security and Surveillance Using Face Recognition", in: Int. Conf. Adv. Comput. Data Sci., Springer, 2020: pp. 293-304.

[12] E. Jose, M. Greeshma, M.T.P. Haridas, et al., "Face recognition based surveillance system using facenet and mtcnn on jetson tx2", in: 2019 5th Int. Conf. Adv. Comput. Commun. Syst., IEEE, 2019: pp. 608-613.

[13] A. Kadam, M. Ghadi, A. Chavan, et al., "Multimodal biometric fusion", Int. J. Eng. Sci. 12554 2017.

[14] O.C. Kurban, T. Yildirim, A. Bilgiç, "A multi-biometric recognition system based on deep features of face and gesture energy image", in: 2017 IEEE Int. Conf. Innov. Intell. Syst. Appl., IEEE, 2017: pp. 361-364.

[15] S. Liu, S. Yao, J. Li, et al., "GIobalFusion: A Global Attentional Deep Learning Framework for Multisensor Information Fusion", Proc. ACM Interactive, Mobile, Wearable Ubiquitous Technol. 4 pp. 1-27, 2020.

[16] Q. Memon, "Multi-Layered Multimodal Biometric Authentication for Smartphone Devices", 2020.

[17] S.K.S. Modak, V.K. Jha, "Multibiometric fusion strategy and its applications: A review", Inf. Fusion. 49 pp. 174-204, 2019.

[18] A.H. Mohsin, A.A. Zaidan, B.B. Zaidan, et al., "Real-time remote health monitoring systems using body sensor information and finger vein biometric verification: A multi-layer systematic 
review", J. Med. Syst. 42 pp. 1-36, 2018.

[19] P. Natarajan, S. Wu, S. Vitaladevuni, et al., "Multimodal feature fusion for robust event detection in web videos", in: 2012 IEEE Conf. Comput. Vis. Pattern Recognit., IEEE, 2012: pp. 1298-1305.

[20] D.T. Nguyen, H.G. Hong, K.W. Kim, et al., "Person recognition system based on a combination of body images from visible light and thermal cameras", Sensors. 17 pp. 605, 2017.

[21] V. Pasquadibisceglie, G. Zaza, G. Castellano, "A personal healthcare system for contact-less estimation of cardiovascular parameters", in: 2018 AEIT Int. Annu. Conf., IEEE, 2018: pp. 1-6.

[22] H. Purohit, P.K. Ajmera, "Optimal feature level fusion for secured human authentication in multimodal biometric system", Mach. Vis. Appl. 32 pp. 1-12, 2021.

[23] J.S. Sahana, R.R. Tarun, C.R. Manjunath, "Multi Biometric Recognition System", Int. J. Comput. Sci. Mob. Comput. 8 pp. 89-94, 2019.

[24] M.A. Said, M. Samuel, A.S. id El-Nafaty, et al., "Novel vision-based thermal people counting tool for tracking infected people with viruses like covid-19", J. Adv. Res. Dyn. Control Syst. 12 pp. 1115-1119, 2020.

[25] M.A. Sánchez-Sánchez, C. Conde, B. Gómez-Ayllón, et al., "Convolutional Neural Network Approach for Multispectral Facial Presentation Attack Detection in Automated Border Control Systems", Entropy. 22 pp. 1296, 2020.

[26] J. Schmidhuber, "Deep learning in neural networks: An overview", Neural Networks. 61 pp. 85$117,2015$.

[27] A. Shajkofci, "Correction of human forehead temperature variations measured by non-contact infrared thermometer", IEEE Sens. J. 2021.

[28] B.G. Vainer, "A novel high-resolution method for the respiration rate and breathing waveforms remote monitoring", Ann. Biomed. Eng. 46 pp. 960-971, 2018. 